\title{
Die Jobinitiative: ein Maßnahmenpaket im Rahmen der stationären medizinischen Rehabilitation zur Förderung der beruflichen Reintegration arbeitsloser Alkohol- und Medikamentenabhängiger
}

U. Zemlin

\author{
The Job Promotion Program - Measures in Indoor Addiction Therapy for \\ Promoting Vocational Reintegration of the Unemployed
}

\section{Zusammenfassung}

Um die Vermittlungschancen arbeitsloser Suchtkranker am Arbeitsmarkt zu erhöhen und deren aktives Bewältigungsverhalten zu fördern, wurde in der Fachklinik Wilhelmsheim (Oppenweiler) ein Maßnahmenpaket implementiert, das bereits während der medizinischen Rehabilitation die beruflichen Reintegrationsaussichten sondieren, Bewerbungskompetenzen der arbeitslosen Rehabilitanden verbessern, Vorarbeiten für eine nahtlose Anschlussaktivität der Arbeitsverwaltung leisten und die Voraussetzungen für eine gezielte Bewerbungsaktivität im unmittelbaren Anschluss an die Rehabilitation schaffen soll. Aufgrund von Indikationsregeln ist gewährleistet, dass Maßnahmen zur beruflichen Reintegration auch in der Konkurrenz mit anderen Therapiemaßnahmen von hoher Priorität einen angemessenen Stellenwert erhalten. Die Auswertung der therapeutischen Leistungen zeigt, dass Arbeitslose zu einem hohen Prozentsatz zentrale Maßnahmen des Jobinitiative-Programms (berufsbiografische Potenzialanalyse, Arbeitsbelastungserprobungen, Bewerbungscoaching) in Anspruch nehmen und zwar umso mehr, je länger ihre Behandlungsdauer angesetzt ist.

\section{Schlüisselwörter}

Alkoholabhängige Arbeitslose · stationäre Rehabilitation · Jobinitiative

\section{Abstract}

To increase chances of vocational integration and to promote active coping behavior of unemployed patients, the treatment center Fachklinik Wilhelmsheim (71570 Oppenweiler, Germany) has implemented a systematic program which already during addiction therapy analyses individual chances of vocational reintegration, improves competences for application, carries out preliminary measures for a smooth transition towards regional job centers and creates preconditions for well-directed application activity during as well as immediately after indoor addiction therapy. Special rules defining therapy priorities guarantee that measures for vocational reintegration receive appropriate status when competing with other therapeutic measures of high priority as well. Evaluation shows that a high percentage of the unemployed take part in central measures of the job promotion program (analysis of the vocational biography and the occupational prospects, trials for testing ability to withstand job strain, coaching in application activities) all the more, the longer their length of treatment is set.

Key words

Alcohol addicted unemployed $\cdot$ inpatient treatment $\cdot$ program in vocational integration 
Zum Stellenwert von Maßnahmen der beruflichen Reintegration in der medizinischen Rehabilitation

Alle bisherigen Erfahrungen in der Behandlung arbeitsloser Suchtkranker und ebenso die Ergebnisse des ARA-Projekts $[1-4]^{1}$ legen folgende Schlussfolgerungen nahe:

Notwendig ist ein frühzeitiges Sondieren von beruflichen und sozialen Perspektiven in der medizinischen Rehabilitation (im Folgenden auch kurz: Reha), um bereits in der Anfangsphase der stationären Behandlung Schwerpunkte setzen zu können, die geeignet sind, insbesondere bei Arbeitslosen die berufliche und soziale Reintegration zu fördern.

Dabei ist angesichts der begrenzten zeitlichen Ressourcen eine frühzeitige Entscheidung über die Therapiestrategie notwendig: Soll auf eine Reintegration in den Arbeitsmarkt gesetzt werden oder auf eine psychosoziale Stabilisierung in Zeiten von Erwerbslosigkeit, d.h. Entwicklung und Vermittlung von zur Erwerbsarbeit alternativen Lebensentwürfen und Unterstützung bei der Realisierung entsprechender persönlich sinngebender und stabilisierender Zielsetzungen? Ersteres verlangt gezielte Fördermaßnahmen zur Rückkehr in den Arbeitsmarkt und das Vermitteln von aktiven Bewältigungskompetenzen für die Stellensuche, Letzteres bedingt eine Konzentration auf soziales Coping und die Fokussierung auf soziale Stützsysteme, soziale Anknüpfpunkte und die Freizeitgestaltung in der Zeit im Anschluss an die Rehabilitation. Beide Zielsetzungen zu erfüllen, ist angesichts des vielfältigen Reha-Auftrages in der Regel nur ansatzweise möglich. Die daher notwendige Richtungsentscheidung setzt eine frühzeitige Einschätzung der Vermittelbarkeit am Arbeitsmarkt im Sinne eines Profilings voraus.

Berufliche Reintegrationsmaßnahmen müssen im Angebot einer Reha-Klinik weiter intensiviert und ausgebaut werden. Dazu gehören:

- frühzeitiges Vorbereiten der Arbeitssuche und Kontaktanbahnung zur Arbeitsverwaltung bereits während der stationären Rehabilitation;

- Arbeitserprobungsmaßnahmen in Betrieben;

- Vermitteln von Kompetenzen für Stellensuche und Bewerbung und

- motivierende Maßnahmen.

In jedem Fall sollte stärker auf den Zeitraum im unmittelbaren Anschluss an die Reha fokussiert werden, weil in dieser Phase besonders bei Arbeitslosen verstärkt Rückfälle drohen [2-4], was voraussetzt, dass Aufgaben und Tätigkeiten für die ersten drei Monate bereits während der Behandlung strukturiert werden, d.h. eine sehr konkrete Reintegrationsplanung und Krisenplanung erfolgt. Wenn aufgrund der Einschätzung des Reha-Teams die berufliche Reintegration in absehbarer Zeit realisierbar erscheint und daher in den Vordergrund der Reha-Zielsetzung rückt, dann müssen bereits während der Behandlung verstärkte Anstrengungen unternommen werden, um die beruflichen Reintegrationschancen für den Teil der arbeitslosen Klientel zu verbessern, der unter Berücksichtigung der regionalen (Teil-)Arbeitsmärkte beruflich integrierbar erscheint.

\footnotetext{
1 Siehe auch den Beitrag von Henkel, Dornbusch, Zemlin in diesem Heft.
}

Dies verweist zunächst auf die Aufgabe, im Sinne einer berufsbiografischen Potenzialanalyse die individuellen Reintegrationschancen auf der Basis individueller (beruflicher) Kompetenzen und Erfahrungen bzw. berufsrelevanter Lebensbedingungen und Ausgangslagen einzuschätzen. Auf der Grundlage dieser Einschätzung sind dann spezifische Maßnahmen zur Unterstützung der individuellen beruflichen Reintegrationsbemühungen anzubieten, die die einzelnen Patienten/Patientinnen in der Konkurrenz um Arbeitsplätze stärken und bei der Stellensuche konkret unterstützen. Diese Maßnahmen sollen insbesondere auch eine geeignete Vorbereitung auf im Anschluss an die medizinische Rehabilitation stattfindende Reintegrationsmaßnahmen der Arbeitsverwaltung leisten, damit in der sensiblen Phase nach Abschluss der Suchtrehabilitation nahtlose Anschlussaktivitäten der Arbeitsverwaltung ermöglicht und Verzögerungen vermieden werden.

\section{Beschreibung des Maßnahmenpakets ,Jobinitiative“}

Wie kann die medizinische Rehabilitation einen Beitrag zur schnelleren und besseren beruflichen Partizipation leisten und die weitergehende Betreuung durch Job-Center und kommunale Arbeitsgemeinschaften (ARGEs) im Vorfeld unterstützen und vorbereiten? Was ist durch bessere Vernetzung erreichbar?

Unter dieser operationalen Fragestellung wurde in der Fachklinik Wilhelmsheim mit Unterstützung eines Bildungsträgers für berufliche Aus- und Weiterbildung (ProTeGe, Greiz, Thüringen) das Maßnahmenpaket „Jobinitiative“ [5] entwickelt, das folgende Zielsetzungen verfolgt:

1. Berufsbiografische Kompetenzbeschreibung und Potenzialanalyse. Da im Zusammenhang mit und verursacht durch die Suchterkrankung bei den Patienten/Patientinnen in Fachkliniken häufig unzureichende berufliche Ausbildungsvoraussetzungen, häufige Stellenwechsel und unterbrochene Erwerbsbiografien vorliegen und dies insgesamt eine realistische Einschätzung der Chancen am Arbeitsmarkt und die Erarbeitung einer realistischen beruflichen Reintegrationsperspektive erschwert, ist es zunächst von vorrangiger Bedeutung, die individuellen beruflichen und lebenssituationsbezogenen Voraussetzungen zu erheben und mit Blick auf ihre berufliche Verwertbarkeit zu ordnen.

2. Hinführung zur Auseinandersetzung mit den Arbeitsmarktanforderungen. Ein Teil der arbeitslosen Klientel hat, ebenfalls bedingt durch die Suchterkrankung, die Auseinandersetzung mit der Entwicklung und Veränderung der Anforderungen des Arbeitsmarktes versäumt oder aufgegeben, so dass häufig diffuse oder unrealistische Einschätzungen der persönlichen Beschäftigungsfähigkeit zu konstatieren sind. Zur Präzisierung und Korrektur bedarf es einerseits aktueller Informationen über Bedingungen regionaler Arbeitsmärkte und neue Bestimmungen im Bereich der Arbeitsverwaltung, andererseits ist von vorrangiger Bedeutung:

3. Herstellen eines Bewusstseins über die persönliche Beschäftigungsfähigkeit. Dies setzt eine differenzierte Auswertung der eigenen beruflichen Kenntnisse und Erfahrungen und ein Bilanzieren von individuellen berufsbezogenen Stärken und Defiziten voraus. Dabei kann auch eine im Rahmen eines betrieblichen Praktikums (Arbeitsbelastungserprobung, kurz: 
$A B E)$ erworbene Referenzerfahrung hilfreich sein, die eigenen Potenziale einzuschätzen und mit den Anforderungen des Erwerbslebens zu vernetzen.

4. Erarbeiten bzw. Vorbereiten von möglichen Integrationsansätzen. Auf der Grundlage der berufsbiografischen Potenzialanalyse und einer fundierten Einschätzung der Beschäftigungsmöglichkeiten müssen geeignete Vorgehensweisen geplant werden, die einen Zugang zu Beschäftigungsmöglichkeiten öffnen, z.B. durch geeignete Bewerbungstechniken und/oder einen niedrigschwelligen Zugang zu Beschäftigung z. B. über Praktika oder Zeitarbeit.

5. Fördern einer positiven Orientierung. Langfristig mit ausschlaggebend für erfolgreiche Bewerbungen ist ein kontinuierliches persönliches Engagement. Grundlage dafür ist eine positive Einstellung zur Erwerbsarbeit, eine stabile Motivation bei der Arbeitssuche auch angesichts der zu erwartenden Frustrationen bei der Stellensuche aufgrund von schleppender Bearbeitung und Ablehnungen sowie die Selbstwirksamkeitsüberzeugung und die erforderliche Frustrationstoleranz, um den Marathonlauf der Stellensuche durchzuhalten, einschließlich der Fähigkeit, Misserfolge nicht an die eigene Unfähigkeit zu attribuieren.

6. Förderung von Eigeninitiative. Da die staatlich organisierte Arbeitsverwaltung derzeit nur eingeschränkt in der Lage erscheint, insbesondere für Problemgruppen am Arbeitsmarkt eine effiziente Stellenvermittlung in einen ohnehin sehr begrenzten Stellenmarkt zu gewährleisten, was insbesondere für Langzeitarbeitslose mit mehreren Vermittlungshemmnissen gilt, ist vor allem Eigeninitiative gefragt. Dazu gehören das Wissen um den Zugang zu Stellenangeboten, Initiative zur Nutzung informeller Informationskanäle, Mut zur Initiativbewerbung und die Kompetenzen zur Selbstvermarktung.

7. Persönliche Alternativen zur Erwerbsarbeit im Sinne eines dennoch sinnerfüllten Lebensvollzuges erschließen. Angesichts der wirtschaftlichen Rahmenbedingungen muss davon ausgegangen werden, dass viele Patienten/Patientinnen auch bei stabilem Engagement ohne Erwerbsarbeit ${ }^{2}$ bleiben. Für diese Gruppen wird es vorrangig wichtig sein, sich auch von der Erwerbsarbeit unabhängige Quellen von Sinnerfüllung und sozialer Anerkennung zu erschließen. Dies setzt eine sorgfältige Analyse der persönlichen Neigungen und der sozialen Bezüge voraus.

\section{Wie können diese Zielsetzungen umgesetzt werden?}

Angesichts der Erfordernisse der beruflichen Reintegration von Arbeitslosen wie systematische Erfassung berufsbiografischer Kompetenzen als Voraussetzung für eine gezielte Stellensuche, Durchhaltevermögen angesichts langwieriger und teilweise schleppender und ineffektiver Stellenvermittlung und rationelles und angemessenes Bewerbungsverhalten rückt zunächst die Motivierung zur Arbeitssuche und die frühzeitige Mobilisierung der Arbeitslosen für die Erarbeitung der notwendigen Voraussetzungen für eine Stellensuche bereits aus der Reha heraus in den Vordergrund. Das setzt eine früh angesetzte Information der Patienten/Patientinnen und eine frühe therapeutische Fokussie- rung in der Rehabilitation auf Inhalte der beruflichen Reintegration voraus (Abb. 1).

Deshalb werden bereits in der ersten Phase der Behandlung, in der Regel in der zweiten bis vierten Woche, 2 Informationsveranstaltungen angesetzt, in der Basisinformationen zum Arbeitsmarkt gegeben, Möglichkeiten der Stellensuche aufgezeigt und die dafür notwendigen Voraussetzungen transparent gemacht werden. Diese beiden einführenden Informationsveranstaltungen „Jobinfo 1 und 2“ sind verpflichtend für alle arbeitslosen Patienten/Patientinnen. Sie beinhalten:

Informationen zum Arbeitsmarkt und Konsequenzen des Arbeitsmarktes für Arbeitsverhältnisse (z.B. Zeitarbeit), die Bedeutung von Schlüsselqualifikationen, Rechte und Pflichten Arbeitsloser angesichts der neuen Gesetzgebung inkl. Zumutbarkeitsregelungen, Möglichkeiten der Arbeitssuche neben den klassischen Wegen „Arbeitsagentur“ und „Zeitung“ wie z. B. Initiativbewerbungen und Stellensuche im Internet, Letzteres mit Verweis auf die nötigen Kenntnisse und die Möglichkeit, entsprechende Fertigkeiten in PC- und Internetkursen in der Klinik zu erwerben sowie eine detaillierte Vorstellung des Jobinitiative-Programms der Fachklinik.

Um die nötigen praktischen Fertigkeiten für die Erstellung von Bewerbungsmappen und für die Stellensuche im Internet zu erwerben, werden PC-Kurse (PC-Basiskenntnisse und Einführung in Word, Aufbaukurs Word, Excel, Internetnutzung) angeboten.

Eine zentrale Funktion der Bestandsaufnahme und der Weichenstellung für anschließende gezielte Bewerbungsaktivitäten hat die berufsbiografische Potenzialanalyse. Im Rahmen von in der Regel 8 zweistündigen Gruppensitzungen werden formelle und weitere Qualifikationen und informelle Kenntnisse und Erfahrungen erfasst und die eigenen Kompetenzen überdacht und bewertet. Dies bezieht sich auf berufliche und fachliche Kompetenzen, berufsübergreifende Kompetenzen und Sozialkompetenz sowie personale Kompetenz (Schlüsselqualifikationen). Die persönlichen Rahmenbedingungen werden ermittelt, wie z. B. die berufsbezogene Lebenssituation, die körperliche Verfassung, die persönliche Mobilitätsbereitschaft, Gehaltsvorstellungen, aber auch die persönlichen Vermittlungshemmnisse. Die Patienten/Patientinnen werden angeleitet, den für sie zutreffenden Arbeitsmarkt zu analysieren und ihre Arbeitsmarktchancen zu prüfen, ihren individuellen Suchraum festzulegen und Stellenangebote (Stellenbörsen) zu sondieren, und sie erhalten Informationen zu Vorgaben der Arbeitsagenturen, z. B. Zumutbarkeitskriterien, überregionale Mobilität, Akzeptanz von Zeitarbeit und geringfügiger Beschäftigung usw. Sie werden angehalten, ihre berufsbezogenen Erwartungen zu überdenken, die Realisierbarkeit der eigenen Berufswünsche zu bewerten, ihre Gehaltsvorstellungen zu überprüfen und gewünschte und realisierbare Arbeitsbedingungen (z.B. Zeitarbeit, Schichtarbeit) abzugleichen. Es erfolgt eine Bilanz der bisher erfolgten und der erforderlichen Bewerbungsaktivitäten. Ein zentraler Baustein ist die Anleitung zur Nutzung des Internets für die Stellensuche über Jobbörsen, die Suche nach Informationen über Berufsbilder bzw. für die Ermittlung von Informationen über Ausbildungen und Fortbildungen.

\footnotetext{
2 Vgl. hierzu Henkel, Grünbeck in diesem Heft.
} 


\begin{tabular}{|c|c|c|c|c|}
\hline \multirow[t]{3}{*}{1} & \multicolumn{3}{|c|}{$\begin{array}{c}\text { Psychosoziale Eingangsdiagnostik } \\
\text { z.B. psychosoziale Anamnese, Berufsanamnese, } \\
\text { EDV-gestützte Testdiagnostik }\end{array}$} & \multirow{4}{*}{$\begin{array}{l}\text { Medizinische } \\
\text { Eingansg- } \\
\text { untersuchung } \\
\text { und } \\
\text { sozialmedizinische } \\
\text { Bewertung }\end{array}$} \\
\hline & \multicolumn{3}{|c|}{ Beruflich relevante Ergebnisse? } & \\
\hline & \multicolumn{3}{|c|}{$\begin{array}{l}\text { Maßnahme: „Jobinfo“ und } \\
\text { Auswertung im Einzelgespräch }\end{array}$} & \\
\hline \multirow[t]{4}{*}{3} & \multicolumn{3}{|c|}{$\begin{array}{l}\downarrow \text { Interdisziplinäre Indikationskonferenz: } \\
\text { Besteht Aussicht auf berufliche Reintegration? }\end{array}$} & \\
\hline & \multirow[t]{2}{*}{ ja } & \multirow{2}{*}{\multicolumn{2}{|c|}{$\begin{array}{l}\text { Information nicht } \\
\text { ausreichend }\end{array}$}} & \multirow{3}{*}{$\begin{array}{l}\text { Medizinischer } \\
\text { Handlungsbedarf? }\end{array}$} \\
\hline & & & & \\
\hline & \multicolumn{3}{|c|}{ Weiterer Klärungsbedarf? } & \\
\hline \multirow[t]{3}{*}{ ab 4} & \multicolumn{3}{|c|}{$\downarrow$ "Berufsbiografische Potenzialanalyse“ } & \multirow{5}{*}{$\begin{array}{c}\text { Weitere } \\
\text { medizinische } \\
\text { Diagnostik } \\
\text { und } \\
\text { medizinische } \\
\text { Behandlung } \\
\text { zur } \\
\text { Wiederherstellung } \\
\text { der Erwerbsfähigkeit }\end{array}$} \\
\hline & \multicolumn{3}{|c|}{$\begin{array}{l}\text { Welche unterstützenden Maßnahmen zur Rückkehr } \\
\text { in den Arbeitsmarkt sind indiziert? }\end{array}$} & \\
\hline & $\begin{array}{l}\text { EDV-Kompetenz } \\
\text { hilfreich oder } \\
\text { notwendig für } \\
\text { Beruf oder für } \\
\text { Bewerbung? } \\
\text { | }\end{array}$ & $\begin{array}{c}\text { Voll- oder teilschichtig } \\
\text { belastbar? Motivierung } \\
\text { durch Betriebspraktikum } \\
\text { sinnvoll? Berufliche } \\
\text { Neuorientierung notwendig? } \\
\text { Berufliche } \\
\text { Versagensängste? } \\
\downarrow\end{array}$ & $\begin{array}{c}\text { Kompetenzen vorhanden } \\
\text { für Stellensuche? } \\
\text { Mündliche od. schriftliche } \\
\text { Bewerbung unterstützen? } \\
\text { Bewerbungsmappe } \\
\text { anlegen? }\end{array}$ & \\
\hline \multirow[t]{2}{*}{ ab 8} & $\begin{array}{l}\text { Maßnahme: } \\
\text { „EDV-Kurse“: } \\
\text { Textverarbeitung } \\
\text { Internet }\end{array}$ & $\begin{array}{l}\text { Maßnahme: } \\
\text { „Arbeitsbelastungs- } \\
\text { erprobung“ (ABE) in } \\
\text { externen Betrieben }\end{array}$ & $\begin{array}{l}\text { Maßnahme: } \\
\text { „Bewerbungscoaching“ }\end{array}$ & \\
\hline & \multicolumn{3}{|c|}{$\begin{array}{l}\text { Sind weitere Maßnahmen sinnvoll? } \\
\text { Therapiedauer ausreichend? }\end{array}$} & \\
\hline \multirow[t]{2}{*}{ ab 12} & $\begin{array}{l}\text { Maßnahme: } \\
\text { „EDV-Kurse“: } \\
\text { Textverarbeitung } \\
\text { Internet }\end{array}$ & $\begin{array}{l}\text { Maßnahme: } \\
\text { „Arbeitsbelastungs- } \\
\text { erprobung“ (ABE) in } \\
\text { externen Betrieben }\end{array}$ & $\begin{array}{l}\text { Maßnahme: } \\
\text { „Bewerbungscoaching“ }\end{array}$ & \multirow{4}{*}{$\begin{array}{l}\text { Ärztliche } \\
\text { Abschluss- } \\
\text { untersuchung }\end{array}$} \\
\hline & \multicolumn{3}{|c|}{$\begin{array}{l}\text { Ist die Einleitung von Kontakten zu weiterbetreuenden Institutionen } \\
\text { notwendig bzw. sinnvoll? }\end{array}$} & \\
\hline $\begin{array}{l}\text { vor- } \\
\text { letzte }\end{array}$ & \multicolumn{3}{|c|}{$\begin{array}{l}\text { Maßnahme: Assistierte Stellenvermittlung } \\
\text { Maßnahme: Kontakt zu Arbeitsagentur bzw. ARGE herstellen }\end{array}$} & \\
\hline letzte & \multicolumn{3}{|c|}{ Reha-Entlassbericht } & \\
\hline
\end{tabular}

Abb. 1 Ablaufschema „Jobinitiative-Programm“.
Während des gesamten Verlaufs erfolgt die Optimierung vorhandener Bewerbungsmappen, die Beurteilung von Arbeitszeugnissen, die Vorbereitung von telefonischen Bewerbungen und gegebenenfalls die Vorbereitung des Vorstellungsgesprächs, wobei einige Inhalte in Einzelgesprächen vertieft werden. Weiterhin werden Informationen zur Existenzgründung, Minijobs, Eingliederungshilfen, privaten Arbeitsvermittlern usw. gegeben.

Als strukturierendes und integrierendes Arbeitsmaterial dient ein Arbeitsheft, das mit Unterstützung eines Bildungsträgers für berufliche Aus- und Weiterbildung (ProTeGe GmbH, Greiz) entwickelt wurde. Das Arbeitsheft ist in einer Diktion formuliert, die mit den in den Agenturen für Arbeit verwendeten Unterlagen kompatibel ist. Es wird bei Behandlungsende von den Patienten/ Patientinnen mitgenommen und kann bei den Agenturen für $\mathrm{Ar}-$ beit vorgelegt und dort für ein Profiling genutzt werden.

Arbeitsbelastungserprobungen (ABE) in externen Betrieben werden in der Regel in einem Zeitrahmen von zwei bis drei Wochen an 4 Arbeitstagen pro Woche durchgeführt. Am jeweils 5. Wochentag erfolgt eine Auswertung der Erfahrungen in der ABEBegleitgruppe. Indikationskriterien für eine Arbeitsbelastungserprobung sind Langzeitarbeitslosigkeit, diffuse berufliche Orientierung oder berufliche Neuorientierung, fragliches vollschichtiges Leistungsvermögen und Ängste vor Rückkehr ins Arbeitsleben
(Versagensängste, soziale Ängste). Am Ende des Praktikums erfolgt ein Bilanzierungsgespräch, um die Erfahrungen mit Blick auf Schlussfolgerungen hinsichtlich beruflicher Orientierung, Belastbarkeit und Motivation zur Stellensuche auszuwerten. Die Ergebnisse werden dem Gruppentherapeuten rückgemeldet. Im Verlauf einer mehrjährigen Aufbauarbeit ist es gelungen, Betriebe in einem breiten Branchenspektrum für die $\mathrm{ABE}$ zu akquirieren, so dass viele Patienten/Patientinnen in berufsnahen Praktika spezifische Erfahrungen sammeln konnten. Als häufigste Erfahrungen seitens der teilnehmenden Patienten/Patientinnen werden genannt: positive Veränderung der Einschätzung des eigenen Leistungsvermögens, Verbesserung der Motivation zur Arbeitssuche, Steigerung von Selbstsicherheit und Selbstwerterleben, positives Erleben sozialer Kontakte am Arbeitsplatz und Konkretisierung des Berufszieles.

Die Zielsetzung der Gruppe Bewerbungscoaching ist darauf gerichtet, das Bewerbungsverhalten zu optimieren, neue Wege der Stellensuche zu erarbeiten, komplette Bewerbungsunterlagen zu erstellen bzw. vorhandene Bewerbungsunterlagen zu vervollständigen und zu optimieren, Vorstellungsgespräche vorzubereiten bzw. Verhalten in Vorstellungsgesprächen zu trainieren und konkrete Bewerbungen bereits während der medizinischen Rehabilitation einzuleiten. Materialien für die Bewerbungsmappe werden von der Klinik zur Verfügung gestellt. Bewerbungsfotos können in der Klinik fertiggestellt werden. 
Die Indikativgruppe „Bewerbungscoaching“ hat folgenden Ablauf, wobei nach jeder Sitzung vorbereitende Hausaufgaben gegeben und assistierend Einzelgespräche durchgeführt werden (Tab. 1):

\section{Tab. 1 Indikativgruppe „Bewerbungscoaching“}

Sitzung 1: Vorstellungsrunde, Aufbau der Bewerbungsmappe, fehlende Unterlagen organisieren;

Sitzung 2: der Lebenslauf - Theorie und Praxis;

Sitzung 3: Bewerbungsfoto, Suche nach Firmen und Stellenangeboten;

Sitzung 4: das Anschreiben - Theorie und Praxis;

Sitzung 5: Erstellen der kompletten Bewerbungsmappe;

Sitzung 6 und 7: Vorbereitung des Vorstellungsgesprächs (telefonisch, persönlich);

Sitzung 8: individuelle Auswertung, weitere Bewerbungen versenden.

Besonders bei Langzeitarbeitslosen, die in den Arbeitsmarkt zurückkehren wollen, ist eine Abfolge mehrerer Jobinitiative-Maßnahmen notwendig (Jobinfo - berufsbiografische Potenzialanalyse - PC-Kurse - ABE - Bewerbungscoaching), die aber aufgrund der fehlenden zeitlichen Ressourcen nicht komplett im Rahmen der stationären Rehabilitation sequenziell abgewickelt werden kann. Deshalb ist es häufig notwendig, Prioritätenentscheidungen $\mathrm{zu}$ treffen und entsprechend die individuell wichtigsten Teile des Gesamtcurriculums zu realisieren.

Um eine optimale Nutzung der Jobinitiative-Angebote $\mathrm{zu}$ gewährleisten, wurde ein Entscheidungsprocedere festgelegt (Abb. 1).

Auf der Grundlage der Befunde der Eingangsuntersuchung, der Berufsanamnese und der medizinischen Befundlage sowie der Auswertung der ersten Einzelgespräche zur Thematik berufliche Integration werden in der interdisziplinären Indikationskonferenz, die der weiteren Behandlungsplanung dient, zusammen mit dem Patienten/der Patientin erste Zuweisungen zu Jobinitiative-Maßnahmen vereinbart. Erweist sich die Befundlage zur Berufsbiografie als noch unzureichend, erfolgt in der Regel die Zuweisung zur berufsbiografischen Potenzialanalyse. Im Anschluss daran können in Abhängigkeit von der Bewilligungsdauer der Reha-Maßnahme adaptiv weitere Indikationsentscheidungen getroffen werden, wobei die Ergebnisse der berufsbiografischen Potenzialanalyse herangezogen werden. Nach Konsultation der Mitarbeiter der Jobinitiative wird vom zuständigen Gruppentherapeuten eingeschätzt, welche weitere Unterstützung zur Vorbereitung der Vermittlung in den Arbeitsmarkt hilfreich bzw. prioritär ist. Entsprechend werden Zuweisungen zu EDV-Kursen, Arbeitsbelastungserprobungen in externen Betrieben oder der Indikativgruppe Bewerbungscoaching mit dem Patienten/der Patientin vereinbart. Im weiteren Verlauf soll eine persönliche Kontaktaufnahme zur Arbeitsagentur oder ARGE erfolgen, um weitere Schritte vorzubereiten.

Als Fortsetzung der genannten Maßnahmen bietet sich angesichts der ungünstigen beruflichen Reintegrationsperspektive arbeitsloser Abhängigkeitskranker an, über das Bewerbungscoaching hinaus direkte Unterstützung im Sinne einer assistierten Stellenvermittlung bereits während der Reha zu organisieren.
Davon könnten Patienten/Patientinnen aus Nord-Württemberg profitieren, da dieser regionale Arbeitsmarkt von der Klinik Wilhelmsheim (nahe Stuttgart) aus erschließbar bzw. zugänglich ist.

\section{Auswertung der Nutzung von Jobinitiative-Maßnahmen}

Um die Nutzung des Jobinitiative-Programms einzuschätzen, wurde ein halber Entlassjahrgang (2. Halbjahr 2004) ausgewertet. Dies betraf 194 arbeitslose Patienten/Patientinnen, davon waren 93 bis 12 Monate arbeitslos und 101 langzeitarbeitslos (über 12 Monate).

Von den bis 12 Monate Arbeitslosen nahmen 32,4\% an der berufsbiografischen Potenzialanalyse und 33,5\% am Bewerbungscoaching teil. Die Teilnahmehäufigkeiten bei den Langzeitarbeitslosen lagen bei 49,6\% und 29,8\%. Hier wird deutlich, dass bei den Langzeitarbeitslosen zunächst Priorität hat, die Berufsbiografie auszuwerten.

Von der berufsbiografischen Potenzialanalyse und/oder dem Bewerbungscoaching wurden $59,1 \%$ der bis 12 Monate Arbeitslosen und 75,2\% der Langzeitarbeitslosen erfasst, so dass davon ausgegangen werden kann, dass trotz eines breit gefächerten Reha-Auftrages die berufliche Reintegration als Zielbereich der Rehabilitation sich bei einem großen Teil der Arbeitslosen durch gezielte Maßnahmen im Behandlungsplan niederschlägt.

In einem weiteren Schritt wurde überprüft, ob die Behandlungsdauer Einfluss auf die Teilnahme an den 3 wichtigsten Maßnahmen des Jobinitiative-Programms hat: berufsbiografische Potenzialanalyse, Arbeitsbelastungserprobung und Bewerbungscoaching. Die Behandlungsdauer wurde entsprechend der häufigsten Bewilligungsdauern fraktioniert in bis 8 Wochen, $>8$ Wochen bis 12 Wochen und $>12$ Wochen.

Die Auswertung ergab, dass die Nutzung der Jobinitiative-Maßnahmen von der Behandlungsdauer abhängt. 32,5\% der Arbeitslosen haben an keiner der zentralen Jobinitiative-Maßnahmen teilgenommen, weil andere therapeutische Maßnahmen als vorrangig bewertet wurden. Dies ist bei bis 8-wöchigen Behandlungsdauern mit 44,7\% häufiger der Fall als bei 8- bis 12-wöchigen Behandlungsdauern (35,7\%) bzw. bei über 12-wöchigen Behandlungsdauern (25,7\%). Die Teilnahme an nur einer der drei zentralen Maßnahmen ist bei allen drei Therapiezeitklassen in etwa gleicher Häufigkeit gewährleistet (44,7\%, 40,5\%, 41,9\%). Zwei oder drei zentrale Jobinitiative-Maßnahmen kamen bei bis 8-wöchigen Behandlungsdauern nur bei 10,6\% zur Anwendung, bei 8 - bis 12-wöchigen Behandlungsdauern in 23,8\% der Fälle, bei über 12-wöchigen Behandlungsdauern hingegen bei 32,4\%. Die Befunde weisen darauf hin, dass bei Arbeitslosen nur eine ausreichend bemessene Behandlungsdauer gewährleisten kann, dass berufliche Reintegrationsmaßnahmen angesichts der sonstigen Behandlungsnotwendigkeiten in angemessenem Ausmaß zur Anwendung kommen können.

Die folgenden Daten zur Arbeitsbelastungserprobung (ABE) und zum Bewerbungscoaching sollen im Sinne einer qualitätssichernden Überprüfung untersuchen, ob zentrale Ziele dieser beiden Maßnahmen erreicht wurden. Dazu wurde jeweils ein größeres 
Zeitfenster gewählt, um aussagekräftige Datenmengen zu gewährleisten.

Für alle Teilnehmer an der ABE aus dem Entlassjahrgang 2004 ( $n=108$ ) wurde untersucht, ob die ABE die notwendige Branchendifferenzierung gewährleisten kann. Die Auswertung ergab, dass in 2004 folgende Branchen genutzt werden konnten: Lager (verschiedene Betriebe): 29, kaufmännischer Bereich (verschiedene Betriebe): 10, Produktion (verschiedene Betriebe): 10, Verkauf (verschiedene Betriebe): 9, Altenpflege: 7, Muster- und Modellbau: 6, einfache Bürotätigkeiten (verschiedene Betriebe): 6, Qualitätssicherung (verschiedene Betriebe): 6, Elektronik (verschiedene Betriebe): 6, Baustoffhandel: 3, Innenausbau: 2, Garten- und Landschaftsbau: 2, Druckerei: 2 sowie Hotelfach, Zahnarztpraxis, Krankenversicherung, Modeatelier, Bauunternehmen, Physiotherapiepraxis, Öffentlichkeitsarbeit, Schaltschrankbau, Werbung, Tierheim: jeweils 1.

Ziel des Bewerbungscoachings ist die Vorbereitung auf bzw. die Initiierung der Stellensuche möglichst bereits während der Suchtbehandlung. Daher wurde bei allen Personen, die in einem Zeitraum von 9 Monaten an einer Indikativgruppe „Bewerbungscoaching“ teilgenommen hatten $(\mathrm{n}=121)$ untersucht, wie häufig sie sich bereits während der Behandlung auf eine Stelle beworben hatten. 56\% der Teilnehmer am Bewerbungscoaching bewarben sich auf dem Arbeitsmarkt, davon 12 auf 1 Stelle, 18 auf 2 Stellen und 38 auf 3 und mehr Stellen. Bei den übrigen 44\% konnte die Zielsetzung nicht umgesetzt werden. Die Gründe dafür werden noch untersucht. Eine erste Einschätzung der zuständigen Mitarbeiter lässt vermuten, dass für einen größeren Teil dieses Personenkreises keine geeigneten Stellen im Internet bzw. in lokalen Printmedien gefunden werden konnten.

\section{Bewertung und Ausblick}

In Anbetracht der restriktiven Arbeitsmarktsituation, der Neuordnung der Arbeitsverwaltung und der insgesamt ungünstigeren Ausgangssituation für arbeitslose Suchtkranke, die in der Regel zahlreiche Vermittlungshemmnisse aufweisen, ist eine systematische Förderung der Teilhabe am Arbeitsleben bereits während der Suchtrehabilitation von vorrangiger Bedeutung.

Das Jobinitiative-Projekt der Fachklinik Wilhelmsheim versucht, zu einer Neugewichtung direkter Reintegrationsmaßnahmen arbeitsloser Suchtkranker in den Arbeitsmarkt im Rahmen der medizinischen Rehabilitation beizutragen. Die Auswertung der ersten Erfahrungen mit diesem Projekt zeigt, dass eine systematische Vorbereitung von direkten Maßnahmen, die der Rückkehr in den Arbeitsmarkt dienen, in der medizinischen Rehabilitation Suchtkranker einen festen Platz finden kann und eine Ausweitung entsprechender Maßnahmen auch angesichts der primären Ziele der Suchtrehabilitation realisierbar ist.

Die Resonanz bei den Patienten/Patientinnen ist positiv. Es gelingt, einen großen Teil der Arbeitslosen in zentrale Maßnahmen wie berufsbiografische Potenzialanalyse, Arbeitsbelastungserprobungen in externen Betrieben sowie Bewerbungscoaching zu integrieren. Die Behandlungsdauer erweist sich allerdings als wesentli- cher Faktor für die Nutzung dieser Behandlungsoptionen. Dies verweist auf die Notwendigkeit, selektive Indikationskriterien und Verfahrensweisen zu entwickeln, die eine Nutzung der vorhandenen Möglichkeiten nach definierten Prioritäten gewährleisten. In der Fachklinik Wilhelmsheim ist dies durch ein internes Indikationsmanual gewährleistet, das rehabilitationsrelevante Befunde erhebt und gewichtet, Prioritätenentscheidungen systematisiert und Vorschläge für die Behandlungsplanung bereitstellt.

Bei einem Teil der behandelten arbeitslosen Suchtkranken ist absehbar, dass die berufliche Reintegrationsperspektive angesichts der genannten Schwierigkeiten trotz der beschriebenen Maßnahmen ungünstig bleiben wird. Hier wäre zu überlegen, ob die Suchtrehabilitation sich bei einer deutlich ungünstigen beruflichen Reintegrationsprognose nicht stärker auf stabilisierende Behandlungselemente konzentrieren sollte, die die betroffenen Patienten/Patientinnen befähigen, ihr Leben auch dann sinnerfüllt und hinreichend befriedigend zu gestalten, wenn eine Rückkehr in den Arbeitsmarkt sich auf absehbare Zeit als nicht umsetzbar erweist.

Eine zentrale Aufgabe für die Zukunft ist es, die im Rahmen berufsreintegrierender Reha-Maßnahmen erzielten Ergebnisse mit den nachfolgenden Aktivitäten der Arbeitsverwaltung, also den Agenturen für Arbeit und den ARGEs, zu vernetzen, um für die Patienten/Patientinnen eine zügige Anschlussaktivität der Arbeitsverwaltung zu ermöglichen. Dafür ist eine Kontaktanbahnung zur Arbeitsverwaltung bereits während der stationären Reha sinnvoll. Einige Leistungsträger tragen dieser Notwendigkeit bereits Rechnung, indem sie die Fahrtkosten dafür übernehmen. Schließlich ist auch die Frage nach einer verstärkten assistierten Stellenvermittlung im Rahmen der Suchtrehabilitation diskussionswürdig. Die Fachklinik Wilhelmsheim plant hierzu für 2006 einen Modellversuch, über den zu einem späteren Zeitpunkt berichtet wird.

\section{Literatur}

${ }^{1}$ Henkel D, Zemlin U, Dornbusch P. Analyse rückfallbeeinflussender Bedingungen bei arbeitslosen Alkoholabhängigen (ARA-Projekt) - Teil I: Einführung in die Thematik, Projektziele, Untersuchungsanlage und Ergebnisse zu Beginn der Suchttherapie. Sucht aktuell 2003; 10 (2): $5-14$

${ }^{2}$ Henkel D, Zemlin U, Dornbusch P. Analyse rückfallbeeinflussender Bedingungen bei arbeitslosen Alkoholabhängigen (ARA-Projekt) - Teil II: Ergebnisse des Therapieverlaufs und der 6-Monats-Katamnese. Sucht aktuell 2004; 11 (1): 21 - 32

${ }^{3}$ Henkel D, Zemlin U, Dornbusch P. Analyse rückfallbeeinflussender Bedingungen bei arbeitslosen Alkoholabhängigen (ARA-Projekt) - Teil III: Abstinenz und Rückfall in der 12-Monats-Katamnese, Veränderungen im Katamneseverlauf, Unterschiede zwischen Rückfälligen und Abstinenten in der Aufnahme-, Entlass- und Katamnesediagnostik sowie zwischen Erst- und Wiederbehandelten. Sucht aktuell 2004; 11 (2): $11-22$

${ }^{4}$ Henkel D, Zemlin U, Dornbusch P. Unemployment and Addiction Therapy. A longitudinal study investigating the abstinence and vocational reintegration chances for alcohol addicted unemployed. In: Kieselbach Th (Hrsg). Unemployment and Health. Bremen: Wissenschaftsverlag, (in press)

${ }^{5}$ Zemlin U. Teilhabe am Arbeitsleben und der Gesellschaft fördern: Empfehlungen und Forderungen aus Sicht der Behandler. In: Fachverband Sucht e.V (Hrsg). Perspektiven für Suchtkranke - Teilhabe fördern, fordern, sichern. Geesthacht: Neuland, 2005: 78-100 